\title{
Reduction and monosegmental fusion for lumbar spondylolisthesis with a long tab percutaneous pedicle screw system: "swing" technique
}

\author{
Bumsoo Park, MD, PhD, ${ }^{1,2}$ Sung-Hyun Noh, MD, ${ }^{1,3}$ and Jeong-Yoon Park, MD1 \\ 1'Department of Neurosurgery, Gangnam Severance Hospital, Spine and Spinal Cord Institute, Yonsei University College of \\ Medicine, Seoul; ${ }^{2}$ Department of Neurosurgery, Chungnam National University Hospital, Chungnam National University School \\ of Medicine, Daejeon; and ${ }^{3}$ Department of Neurosurgery, National Health Insurance Service Ilsan Hospital, Goyang-si, Korea
}

\begin{abstract}
OBJECTIVE With the development of minimally invasive procedures, percutaneous pedicle screw systems have been used to attempt to correct spondylolisthesis. No previous studies have reported on reduction measures using long tab percutaneous pedicle screws for spondylolisthesis. Additional intraoperative correction has been proposed with the "swing" technique. This study was conducted to compare the efficacy of standard minimally invasive transforaminal interbody fusion (MIS-TLIF) with the efficacy of MIS-TLIF with the "swing" technique (MIS-TLIF and swing) in lumbar spondylolisthesis

METHODS This was a matched-control study and included 30 consecutive patients who were followed up for 6 months after surgery. Of those patients, 15 were treated with operative reduction via MIS-TLIF with the "swing" technique, whereas the other 15 were treated with the standard MIS-TLIF procedure. The swing technique is a new reduction procedure for use with long tab percutaneous screws. In the swing technique, the entire system is swung back and forth several times after all constructs are placed. Only patients with Meyerding grade I or II lumbar spondylolisthesis were included in the study (18 with grade I and 12 with grade II). Perioperative and clinical outcomes, radiological parameters (Meyerding grade, percentage of slip, slip correction rate, segmental lordosis, and lumbar lordosis) were compared between groups at 6 months after surgery.

RESULTS Demographic data did not differ significantly between the 2 groups. Postoperative clinical outcomes showed significant improvement in both groups. Postoperative radiological parameters showed that the degree of spondylolisthesis (swing: $4.7 \%$ vs standard: $8.9 \%$ ) and reduction rate (swing: $77.3 \%$ vs standard: $57.1 \%$ ) favored the swing group. The swing technique effectively decreased the degree of spondylolisthesis (swing: $24.1 \%$ to $4.7 \%$ vs standard: $21.8 \%$ to $8.9 \%$ ). No complications related to the procedure were reported.
\end{abstract}

CONCLUSIONS MIS-TLIF with the "swing" technique with long tab percutaneous pedicle screws is a safe and effective reduction method for monosegmental spondylolisthesis. This technique cannot only alleviate symptoms but also achieve nearly completely reduction of slippage.

https://thejns.org/doi/abs/10.3171/2019.2.FOCUS18724

KEYWORDS minimally invasive; transforaminal lumbar interbody fusion; spondylolisthesis; monosegmental fusion; swing technique

$\mathrm{R}$ EDUCTION techniques to restore sagittal balance in patients with spondylolisthesis have been extensively described in the literature over the past decade. A classic reduction method is to attach temporary instruments to an adjacent segment to distract and pull back the slipped segment and then perform monosegmental fusion at the level of spondylolisthesis. ${ }^{15,17}$ While this method is an excellent way to correct spondylolisthesis, it involves instrumenting non-slipped segments, even if only temporarily.

More recently a minimally invasive transforaminal interbody fusion (MIS-TLIF) monosegmental reduction technique that does not require instrumenting non-slipped segments was described by Rajakumar et al., who used a "rocking" technique with a classical constrained percutaneous screw and rod system (Sextant; Medtronic, Sofamor

ABBREVIATIONS BMI = body mass index; EBL = estimated blood loss; MIS-TLIF = minimally invasive transforaminal interbody fusion; PT = pelvic tilt; SS = sacral slope; VAS = visual analog scale.

SUBMITTED December 26, 2018. ACCEPTED February 19, 2019.

INCLUDE WHEN CITING DOI: 10.3171/2019.2.FOCUS18724. 


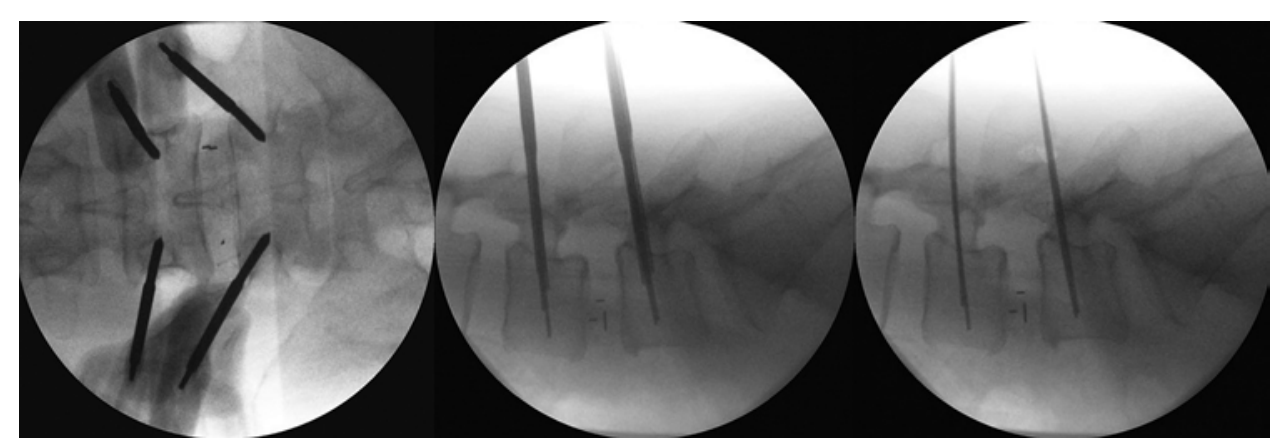

FIG. 1. C-arm fluoroscopic images obtained during the swing technique showing insertion of Jamshidi needles into the pedicles at the level of interest and passing of K-wires through the Jamshidi needles.

Danek) for grade II and higher grades of lumbar spondylolisthesis. ${ }^{13}$ Recently, a long tab type percutaneous screw system was developed and has come into common use because of its simplicity and similarity to conventional pedicle screw systems. To date, however, there have been no reports about reduction measures using long tab percutaneous pedicle screw for spondylolisthesis.

A "swing" technique was developed as a modified version of the rocking technique to be more operator friendly. The technique was used with a long tab percutaneous pedicle screw and rod system for better and easier manipulation in the last step of the surgery. The purpose of this report is to assess the efficacy of the "swing" technique by comparing it with conventional MIS-TLIF for reducing single-level lumbar spondylolisthesis.

\section{Methods \\ Clinical Data}

This was a retrospective, matched-control study of 30 consecutive patients who underwent minimally invasive surgery for monosegment spondylolisthesis and were followed up for 6 months. The surgeries were performed in 2017 and 2018 by a single surgeon (J.Y.P.) using a single, long, PEEK (polyetheretherketone), interbody bulletshaped cage (Capstone; Medtronic, Sofamor Danek) and percutaneous pedicle screw and rod system (Zenius; Medyssey). This study was approved by the institutional review board of Gangnam Severance Hospital, Yonsei University College of Medicine, and patients provided consent to trial participation. The patients' mean age was $63.1 \pm 9.9$ years. Twenty of the patients had degenerative spondylolisthesis and 10 had isthmic spondylolisthesis. Fifteen patients underwent single-level MIS-TLIF with the "swing" technique, and the other 15 patients underwent standard MISTLIF. All patients in both groups presented with low-back pain with varying degrees of radiating pain or neurological symptoms. All patients had conservative treatment before surgical treatment was considered. Exclusion criteria were pathological conditions such as spinal malignancy, infection, or trauma.

\section{Surgical Techniques}

Each patient was placed on a Jackson surgical table in the prone position after induction of general anesthesia.
The MIS-TLIF procedure was performed on the more symptomatic side. The level of interest was confirmed with C-arm fluoroscopy. A horizontal line was drawn on the skin at the disc space and facet joint, and the lateral margins of the pedicles were marked on each side. A 2.5to 3-cm-long paramedian incision was made at the lateral margins of the pedicles, centered on disc space bilaterally. ${ }^{3}$ Intraoperative neurophysiological monitoring was not used in this study because all patients had only grade I or II spondylolisthesis.

Sequential tubular dilators (22-mm diameter; MetRx, Medtronic Sofamor Danek) were inserted through the incision to dock onto the facet joint. A tubular retractor was fixed to a flexible arm that was attached to the side of the table. The level was reconfirmed with C-arm fluoroscopy. Under a microscope, muscles were dissected to expose the facet joint. Facetectomy was performed with a high-speed drill and chisel osteotome. The inferior and superior articular processes were chiseled enough to gain access to the disc space and free the nerve root but not so much as to damage the pedicle. The ligamentum flavum was resected with a Kerrison punch, and the contralateral side was decompressed for central canal stenosis. After the thecal sac and nerve roots were identified, total discectomy was performed. After gently retracting the thecal sac together with the root, the annulus was incised with a no. 15 blade. The disc was removed with various punching tools and shavers, and the endplate was curetted with a down-biting curette to remove cartilaginous material. After extensive decompression, including decompression of the central stenosis and contralateral side, the space was packed with autologous local bone obtained from the articulating processes. A TLIF cage filled only with autologous local bone was placed at the level. After interbody fusion, the tubular retractor was removed. ${ }^{3}$

Under fluoroscopic guidance, percutaneous pedicle screw-rod constructs were placed bilaterally. Jamshidi needles were inserted into the pedicles, and K-wires were passed through the Jamshidi needles (Fig. 1). Cannulated tappers and screws were used to advance the K-wires. Rods were placed percutaneously to connect the screws. After the construct, including the caps, was inserted, the screws were tightened but not locked to retain screw head movement and loosened half a turn from proper tightening (Fig. 2). All screw tabs and rod holders were gently swung back and forth in a craniocaudal direction several 


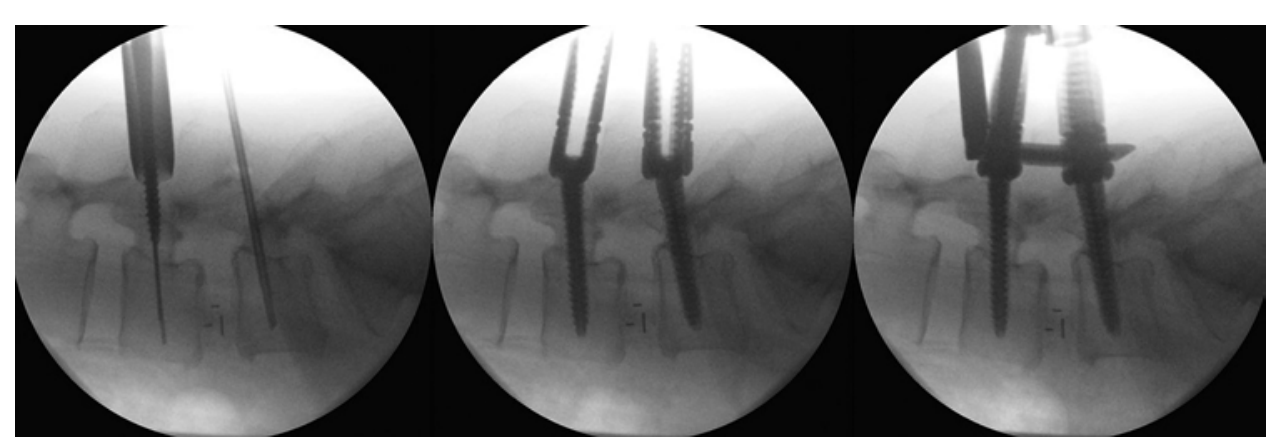

FIG. 2. C-arm fluoroscopic images obtained during the swing technique showing advancement of cannulated tappers and screws over the K-wires and placement of the percutaneous pedicle screw-rod construct (Zenius) bilaterally.

times to find the right reduction angle under continuous fluoroscopic guidance (Figs. 3 and 4 and Video 1).

VIDEO 1. Video clip demonstrating the swing technique. The technique is performed by swinging back and forth in a craniocaudal direction while holding all the screw tabs and rod holders. After finding the right reduction angle by means of the swing technique with continuous fluoroscopic guidance, all towers and rod holders are held in position, and the final tightening the caps is performed. Copyright Gangnam Severance Hospital. Published with permission. Click here to view.

After finding the reduction angle, all towers and rod holders were held in position during final tightening of the caps (Fig. 5, Video 1). Postoperatively, 6-month follow-up lateral radiographs showed good reduction and fusion of spondylolisthesis between L4 and L5 (Fig. 6).

\section{Clinical and Radiological Evaluation}

Age, sex, body mass index (BMI), and bone mineral density were collected. Clinical results were quantified by visual analog scale (VAS) pain scores obtained preoperatively and at the 6-month follow-up. Radiological evaluation included slip percentage, slip correction rate, segmental lordosis, and lumbar lordosis preoperatively and at the 6-month follow-up. Sagittal parameters were measured with whole-spine plain radiographs. Slip percentage was measured as a percentage of the distance from the posterior border of the caudal vertebra to the posterior border of the rostral vertebra. ${ }^{11}$ Slip correction rate was calculated as: [(preoperative - postoperative slip percentage)/(preoperative slip percentage) $\times 100 .{ }^{12}$ All clinical and radiological parameters were compared between the swing and standard groups. Comparison between preoperative and postoperative VAS scores was performed for both groups.

\section{Statistical Analysis}

Statistical analysis was performed with SPSS Statistics for Windows, version 23 (IBM Corp.). Mann-Whitney U-tests and Wilcoxon tests were used to compare clinical and radiological outcomes between groups; $p$ values below 0.05 were defined as statistically significant.

\section{Results}

A total of 30 consecutive patients with spondylolisthesis underwent MIS-TLIF. Of the 30 patients, $18 \mathrm{had}$ Meyerding grade I and 12 had Meyerding grade II spondylolisthesis. Fifteen patients underwent MIS-TLIF with the swing technique, and the other 15 patients underwent
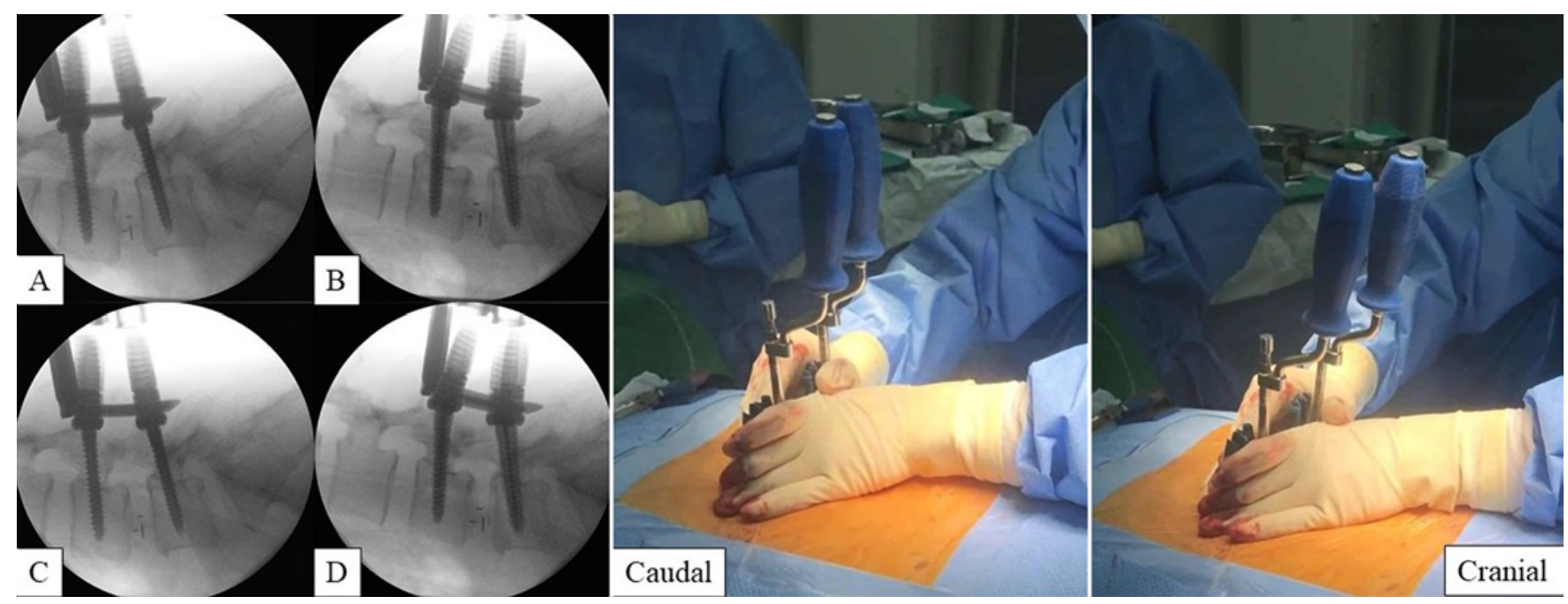

FIG. 3. Left: Sequential fluoroscopic images (showing all towers of the screws with the rod holders being gently swung back and forth to find the right reduction angle. Center and right: Photographs of surgeon performing the swing technique. 


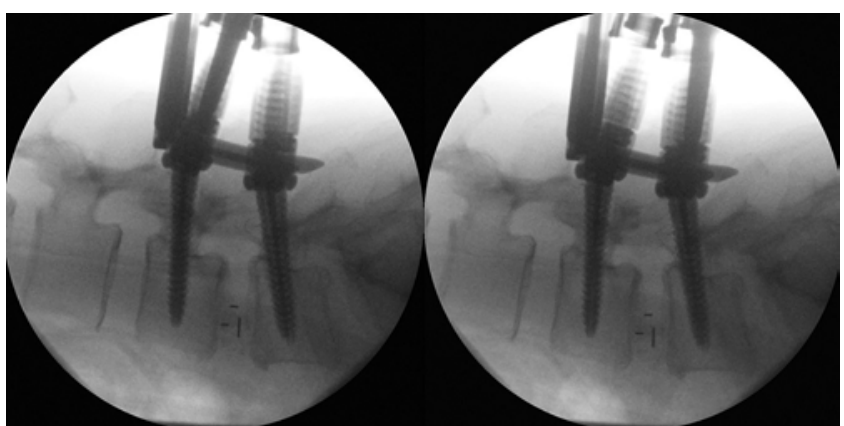

FIG. 4. C-arm fluoroscopic images obtained during the swing technique. Tightening of the caps at the reduction angle.

standard MIS-TLIF as a control. Demographic and baseline clinical characteristics of the patients in the 2 groups are summarized in Table 1. The preoperative level and types of spondylolisthesis were similar in both groups. Both groups had similar sex and age distributions. No major complications associated with the operation were reported. The mean operative time (from skin incision to closure) was 115.3 minutes, and the mean estimated blood loss (EBL) was approximately $267 \mathrm{ml}$. All patients were discharged on the 11th postoperative day. Table 2 summarizes the perioperative data for the swing and standard groups.

\section{Clinical Outcomes}

At the 6-month follow-up evaluation, most of the 15 patients who received the "swing" technique were pain free and the remaining patients in this group had minimal pain. The mean preoperative VAS score was $6.9 \pm$ 1.6, whereas the mean score at the last follow-up was 2.9 \pm 2.4 . All patients had significant improvement in pain scores after treatment in both groups $(\mathrm{p}<0.05)$. The mean pain scores did not differ significantly between the swing and standard groups (Table 2). There were no operationrelated complications in either group.

\section{Radiological Outcomes}

MIS-TLIF with the "swing" technique nearly completely reduced all grades of spondylolisthesis. The percentage of slip in the swing group decreased dramatically from $24.1 \% \pm 7.3 \%$ to $4.7 \% \pm 5.3 \%$ postoperatively ( $p<$ $0.05)$. The percentage of slip decreased in the standard group from $21.8 \% \pm 7.0 \%$ preoperatively to $8.9 \% \pm 5.8 \%$ postoperatively. The preoperative slip for the swing and standard groups did not differ significantly, but there was a significant reduction from the preoperative percentage of slip ( $\mathrm{p}<0.05)$. Segmental lordosis and lumbar lordosis did not differ significantly between groups. Table 2 summarizes the radiological data for the swing and standard groups, and representative surgical cases are shown in Fig. 6.

\section{Discussion}

Classic methods to restore sagittal balance for spondylolisthesis involve temporarily instrumenting an adjacent segment for distraction and reduction. ${ }^{15}$ However, use of a

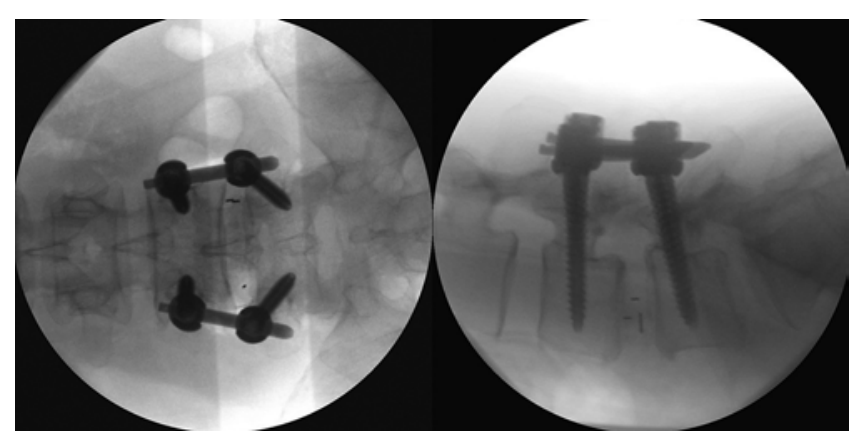

FIG. 5. C-arm fluoroscopic images of the construct in place after breaking the towers.

normal segment to pull back the slipped segment can be avoided by monosegmental reduction with the MIS-TLIF technique. A search of the literature revealed that MISTLIF can be used to treat low-grade spondylolisthesis with moderately good clinical results. ${ }^{8,10,13}$ Some of the many advantages of MIS-TLIF include smaller skin incisions, reduced muscle disruption and denervation, less handling of nerves, and decreased blood loss, while providing good to excellent fusion results. ${ }^{7,14,19,20}$

The question of whether monosegmental spondylolisthesis requires reduction is controversial. However, sagittal alignment plays an important role in the development of many spinal disorders. ${ }^{1,4}$ Correcting spondylolisthesis is desirable for the effects of indirect decompression of the neuroforamina and restoration of spinal sagittal balance. Restored spinal sagittal balance was shown by Bourghli et al. to correlate with good clinical outcomes following surgery for low-grade spondylolisthesis. ${ }^{2,18}$ They found significant improvement toward normal pelvic tilt (PT) and sacral slope (SS) values in relation to pelvic incidence (PI) after surgery. Reduction combined with fusion of the slipped vertebra could correct anterior tilt with anterior sagittal imbalance due to spondylolisthesis, thus restoring a normal spinosacral angle. For patients who did not have sufficiently restored sagittal balance parameters, outcomes were not as good as for patients who had restored sagittal balance. Thus surgical management of spondylolisthesis that restores pelvic position-dependent parameters showed good clinical outcomes. ${ }^{2,13}$ Martiniani et al. suggested reducing slippage for patients with highgrade spondylolisthesis and an unbalanced pelvis because spinopelvic parameters, such as PT, SS, and LL, could be better corrected. ${ }^{9,16}$ This result indicates that reducing slippage would be beneficial for relieving low-back pain, reducing disability and improving quality of life in patients who have an unbalanced pelvis preoperatively. ${ }^{16}$ Most authors, including Ruf et al., ${ }^{15}$ Floman et al., ${ }^{5}$ and Goyal et al., ${ }^{6}$ have promoted reducing spondylolisthesis as far as possible. These studies indicate that reduction can restore lumbar alignment and increase the contact area between the slipped and inferior vertebral bodies, which benefits bone graft fusion. ${ }^{16}$

In the present study, some values seem to show more favorable outcome for the standard group compared with the swing group. At 6 months after surgery, the VAS pain score was lower in the standard group than in the swing 


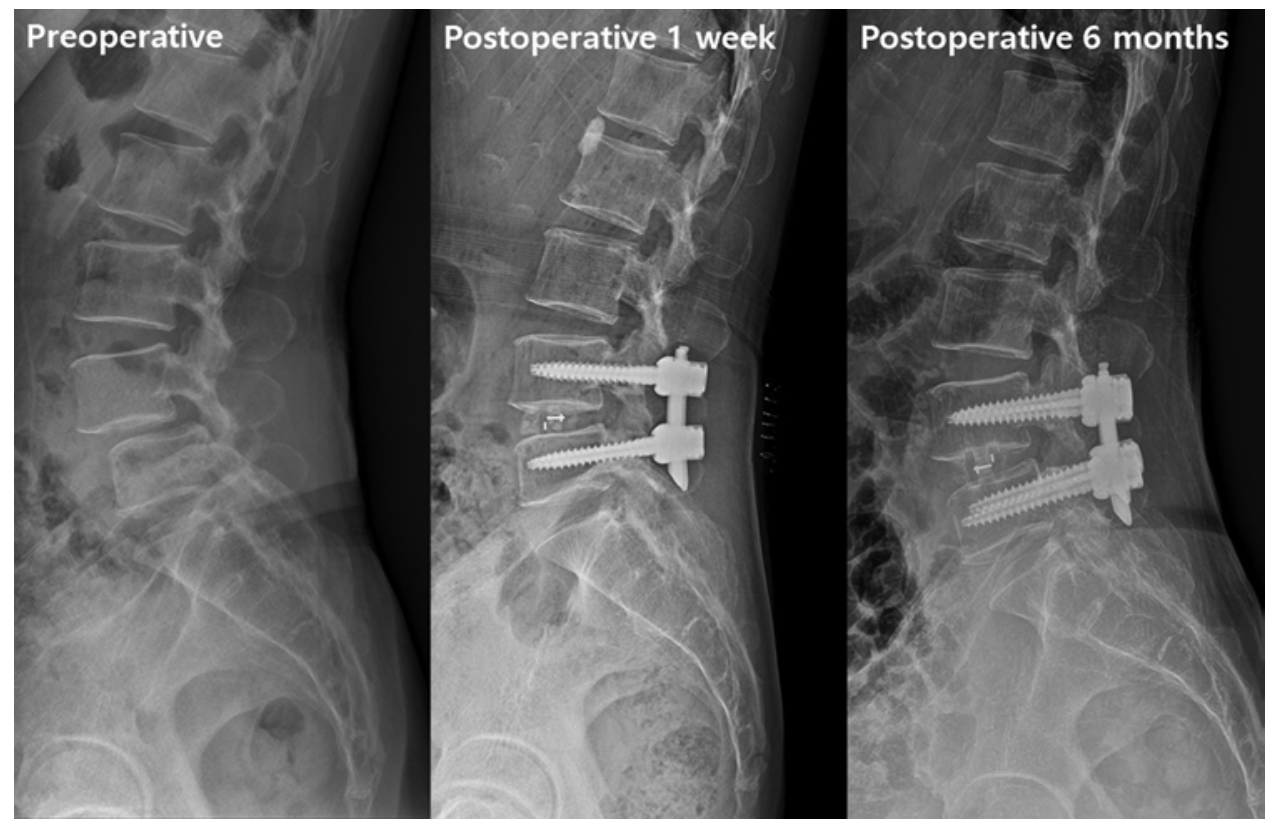

FIG. 6. Radiographs of the lumbar spine of a 54-year-old male patient with degenerative spondylolisthesis showing good reduction of slippage at 1 week after surgery and good fusion 6 months after surgery.

group (1.5 vs 2.9), although the difference was not statistically significant. The higher slip correction rate in the swing group (77\% vs $57 \%$ in the standard group) might have caused somewhat unfavorable early clinical results. The stronger correction force in the patients who had been operated on with the swing technique could have acted as a pain source. In addition, although not significant, 6-month postoperative lumbar lordosis $\left(43^{\circ}\right.$ vs $\left.48.8^{\circ}\right)$ was also a little higher in the standard group. Because preoperative lumbar lordosis $\left(41.5^{\circ}\right.$ vs $\left.46.3^{\circ}\right)$ was higher in the standard group, direct comparison could be misleading (Table 2). To determine the impact of the sagittal balance after spondylolisthesis correction by swing technique, more long-term follow-up with a larger sample size is needed.

Although the "rocking" technique is effective, the nature of the Sextant system (CD Horizon Sextant; Medtronic), onto which the towers are mated and to which the arc has to be attached to insert the precurved lordotic rod into the screw heads, makes it difficult to handle. To overcome this problem, a long tab percutaneous pedicle screw system was developed. With this system, placing the rod into the screw heads is easy, and holding all the long tabs and rod holders together on each side helps deliver the swinging motion to the slip segments. This motion helps realign the sagittal profile. The procedure was carried out at the end of the operation to avoid disruption of flow. It is safe, simple, and easy to perform. According to this study, the "swing" technique fulfills MIS-TLIF by reducing slippage. The surgical times for the "swing" technique and conventional MIS-TLIF did not differ much. With a few minutes of attention and little effort, slippage can be corrected with the "swing" technique.

When to insert the cage-before or after screw placement-is a matter to consider. In conventional fusion surgery, screws can be installed before implanting the cage for distraction and reduction. However, for MIS-TLIF, which uses a tubular retractor, it is almost impossible to insert the cage after pedicle screw placement, because of the small skin incision ( 2.5 to $3 \mathrm{~cm}$ ). Of course, placing a cage after contralateral pedicle screw insertion is possible, but it is hard to obtain enough reduction force with unilateral screws. To get enough reduction force, insert-

TABLE 1. Demographic and baseline clinical characteristics

\begin{tabular}{|c|c|c|}
\hline \multirow[b]{2}{*}{ Characteristic } & \multicolumn{2}{|c|}{ Group } \\
\hline & Swing & Standard \\
\hline No. of patients & 15 & 15 \\
\hline Age (yrs), mean & $63.8 \pm 6.4$ & $63.5 \pm 10.0$ \\
\hline \multicolumn{3}{|l|}{ Sex } \\
\hline M & 3 & 4 \\
\hline $\mathrm{F}$ & 12 & 11 \\
\hline BMI $\left(\mathrm{kg} / \mathrm{m}^{2}\right)$, mean & $23.7 \pm 2.7$ & $25.4 \pm 3.5$ \\
\hline Osteoporosis (T-score $\leq 2.5$ ) & $5(33.3 \%)$ & $5(33.3 \%)$ \\
\hline \multicolumn{3}{|l|}{ Level of slippage } \\
\hline L3-4 & 1 & 2 \\
\hline L4-5 & 10 & 10 \\
\hline L5-S1 & 4 & 3 \\
\hline \multicolumn{3}{|l|}{ Spondylolisthesis grade } \\
\hline I & 8 & 10 \\
\hline$\|$ & 7 & 5 \\
\hline \multicolumn{3}{|l|}{ Type of spondylolisthesis } \\
\hline Isthmic & 6 & 4 \\
\hline Degeneration & 9 & 11 \\
\hline
\end{tabular}

Data are numbers of patients unless otherwise indicated. Mean values are presented with SDs. 
TABLE 2. Comparison of perioperative and radiologic data between groups

\begin{tabular}{|c|c|c|c|}
\hline \multirow[b]{2}{*}{ Variable } & \multicolumn{2}{|c|}{ Group } & \multirow{2}{*}{$\begin{array}{c}p \\
\text { Value* }\end{array}$} \\
\hline & Swing & Standard & \\
\hline Operating time (mins), mean & $115.3 \pm 38.3$ & $114.6 \pm 36.4$ & 0.48 \\
\hline EBL, mean & $267.3 \pm 158.8$ & $276 \pm 180.3$ & 0.71 \\
\hline \multicolumn{4}{|l|}{ VAS } \\
\hline Preop & $6.9 \pm 1.6$ & $6.9 \pm 0.9$ & 0.90 \\
\hline Postop & $2.9 \pm 2.4$ & $1.5 \pm 1.3$ & 0.68 \\
\hline \multicolumn{4}{|l|}{ Slip (\%)† } \\
\hline Preop & $24.1 \pm 7.3$ & $21.8 \pm 7.0$ & 0.32 \\
\hline 6 mos postop & $4.7 \pm 5.3$ & $8.9 \pm 5.8$ & 0.04 \\
\hline $\begin{array}{l}\text { Slip correction rate }(\%) \text {, } \\
\text { mean } \ddagger\end{array}$ & $77.3 \pm 29$ & $57.1 \pm 28.1$ & 0.05 \\
\hline Complications & 0 & 0 & \\
\hline \multicolumn{4}{|l|}{ Segmental lordosis } \\
\hline Preop & $12.3 \pm 8.5$ & $11.5 \pm 7.9$ & 0.93 \\
\hline 6 mos postop & $14.5 \pm 8.7$ & $12.4 \pm 5.5$ & 0.68 \\
\hline \multicolumn{4}{|l|}{ Lumbar lordosis } \\
\hline Preop & $41.5 \pm 9.1$ & $46.3 \pm 10.5$ & 0.25 \\
\hline 6 mos postop & $43.0 \pm 10.5$ & $48.8 \pm 7.8$ & 0.14 \\
\hline
\end{tabular}

$\mathrm{EBL}=$ estimated blood loss; mins = minutes.

Data are presented as mean values and SDs. Boldface type indicates statistical significance.

* Mann-Whitney U-test.

† Slip (\%) measured as a percentage of the distance from the posterior border of the caudal vertebra to the posterior border of the rostral vertebra.

$\ddagger$ Slip correction rate $(\%)=[($ preoperative - postoperative slip percentage $) /$

(preoperative slip percentage) $\times 100]$.

ing the cage before placing bilateral percutaneous screws was practiced for this swing technique. Since there were no procedure-related complications, such as subsidence, endplate damage, or cage breakage, it is safe to say that inserting the cage first would not be a problem.

\section{Study Limitations}

This study has limitations. The sample size was small, and the follow-up period was short for evaluation of fusion rates. However, in an earlier study, the senior author (J.Y.P.) had already reported an acceptable fusion rate for MIS-TLIF. ${ }^{3}$ Because there was no difference in the use of instruments and fusion materials except for the addition of the swing technique, the fusion rate would not be expected to be affected. However, to reduce variability, further studies should include a larger sample size and longer followup. To fully assess the efficacy of the technique, clinical and radiological outcomes must be further evaluated, with measurement of sagittal parameters and fusion rates.

\section{Conclusions}

This study was conducted to assess the effectiveness of the "swing" technique. The clinical and radiological outcomes of standard MIS-TLIF and MIS-TLIF with the "swing" technique were compared. Spondylolisthesis was significantly restored and corrected with the "swing" tech- nique. Minimally invasive monosegmental TLIF with the "swing" technique is effective, safe, and easy to perform.

\section{References}

1. Bernhardt M, Bridwell KH: Segmental analysis of the sagittal plane alignment of the normal thoracic and lumbar spines and thoracolumbar junction. Spine (Phila Pa 1976) 14:717721, 1989

2. Bourghli A, Aunoble S, Reebye O, Le Huec JC: Correlation of clinical outcome and spinopelvic sagittal alignment after surgical treatment of low-grade isthmic spondylolisthesis. Eur Spine J 20 (Suppl 5):663-668, 2011

3. Choi UY, Park JY, Kim KH, Kuh SU, Chin DK, Kim KS, et al: Unilateral versus bilateral percutaneous pedicle screw fixation in minimally invasive transforaminal lumbar interbody fusion. Neurosurg Focus 35(2):E11, 2013

4. Farfan HF, Huberdeau RM, Dubow HI: Lumbar intervertebral disc degeneration: the influence of geometrical features on the pattern of disc degeneration-a post mortem study. $\mathbf{J}$ Bone Joint Surg Am 54:492-510, 1972

5. Floman Y, Millgram MA, Ashkenazi E, Smorgick Y, Rand $\mathrm{N}$ : Instrumented slip reduction and fusion for painful unstable isthmic spondylolisthesis in adults. J Spinal Disord Tech 21:477-483, 2008

6. Goyal N, Wimberley DW, Hyatt A, Zeiller S, Vaccaro AR, Hilibrand AS, et al: Radiographic and clinical outcomes after instrumented reduction and transforaminal lumbar interbody fusion of mid and high-grade isthmic spondylolisthesis. J Spinal Disord Tech 22:321-327, 2009

7. Hari A, Krishna M, Rajagandhi S, Rajakumar DV: Minimally invasive transforaminal lumbar interbody fusion-indications and clinical experience. Neurol India 64:444-454, 2016

8. Kim JS, Jung B, Lee SH: Instrumented minimally invasive spinal-transforaminal lumbar interbody fusion (MIS-TLIF): minimum 5-year follow-up with clinical and radiologic outcomes. Clin Spine Surg 31:E302-E309, 2018

9. Martiniani M, Lamartina C, Specchia N: "In situ" fusion or reduction in high-grade high dysplastic developmental spondylolisthesis (HDSS). Eur Spine J 21 (Suppl 1):S134-S140, 2012

10. Mummaneni PV: Percutaneous transforaminal lumbar interbody fusion for the treatment of degenerative lumbar instability. Neurosurgery 62:E1384, 2008

11. Oh CH, Ji GY, Jeon JK, Lee J, Yoon SH, Hyun DK: Slip reduction rate between minimal invasive and conventional unilateral transforaminal interbody fusion in patients with low-grade isthmic spondylolisthesis. Korean J Spine 10:232-236, 2013

12. Omidi-Kashani F, Hassankhani EG, Shiravani R, Mirkazemi M: Surgical outcome of reduction and instrumented fusion in lumbar degenerative spondylolisthesis. Iran J Med Sci 41:13-18, 2016

13. Rajakumar DV, Hari A, Krishna M, Sharma A, Reddy M: Complete anatomic reduction and monosegmental fusion for lumbar spondylolisthesis of Grade II and higher: use of the minimally invasive "rocking" technique. Neurosurg Focus 43(2):E12, 2017

14. Rampersaud YR, Gray R, Lewis SJ, Massicotte EM, Fehlings MG: Cost-utility analysis of posterior minimally invasive fusion compared with conventional open fusion for lumbar spondylolisthesis. SAS J 5:29-35, 2011

15. Ruf M, Melcher R, Merk H, Harms J: [Anatomic reduction and monosegmental fusion for high-grade developmental spondylolisthesis L5/S1.] Z Orthop Ihre Grenzgeb 144:33-39, 2006 (German)

16. Shi L, Chen Y, Miao J, Shi J, Chen D: Reduction of slippage influences surgical outcomes of grade II and III lumbar isth- 
mic spondylolistheis. World Neurosurg 120:e1017-e1023, 2018

17. Sudarshan PK, Suthar HR, Varma VK, Krishnan A, Hegde SK: Long-term experience with reduction technique in high-grade spondylolisthesis in the young. Int J Spine Surg 12:399-407, 2018

18. Tay KS, Bassi A, Yeo W, Yue WM: Intraoperative reduction does not result in better outcomes in low-grade lumbar spondylolisthesis with neurogenic symptoms after minimally invasive transforaminal lumbar interbody fusion-a 5-year follow-up study. Spine J 16:182-190, 2016

19. Wang J, Zhou Y, Zhang ZF, Li CQ, Zheng WJ, Liu J: Comparison of one-level minimally invasive and open transforaminal lumbar interbody fusion in degenerative and isthmic spondylolisthesis grades 1 and 2. Eur Spine J 19:1780-1784, 2010

20. Wu JC, Mummaneni PV: Using lumbar interspinous anchor with transforaminal lumbar interbody fixation. World Neurosurg 73:471-472, 2010

\section{Disclosures}

The authors report no conflict of interest concerning the materials or methods used in this study or the findings specified in this paper.

\section{Author Contributions}

Conception and design: JY Park. Acquisition of data: all authors. Analysis and interpretation of data: all authors. Drafting the article: JY Park, B Park. Critically revising the article: JY Park. Reviewed submitted version of manuscript: JY Park, B Park. Approved the final version of the manuscript on behalf of all authors: JY Park. Statistical analysis: B Park. Study supervision: JY Park, Noh.

\section{Supplemental Information}

Videos

Video 1. https://vimeo.com/322767136.

\section{Correspondence}

Jeong-Yoon Park: Gangnam Severance Hospital, Spine and Spinal Cord Institute, Yonsei University College of Medicine, Seoul, Korea.spinepjy@gmail.com. 